\title{
ELECCIONES PRESIDENCIALES PRIMARIAS EN ESTADOS UNIDOS
}

En el Estado de Puerto Rico tuvieron lugar el pasado 17 de febrera de 1980 las elecciones presidenciales primarias para el nombramiento de los delegados del partido republicano que iban a asistir a la Convención Nacional en la que se designaría el candidato a la presidencia por aquel partido. Desde esa fecha hasta el 3 de junio de 1980 se han celebrado elecciones primarias en un total de treinta y cuatro Estados, además de en Puerto Rico ${ }^{1}$ y el distrito de Columbia ${ }^{2}$.

Una mirada retrospectiva permite constatar que 1980 es el año en el que mayor número de elecciones primarias se han celebrado. Según parece, en 1968 quince Estados celebraron elecciones presidenciales primarias de uno u otro tipo; en 1972, el número de primarias ascendió a veintidós, y en 1976, a treinta.

Sean cuales fueren las razones que motivan este incremento progresivo, lo cierto es que tal hecho significa, cuando menos, un aumento de la representatividad popular de los delegados asistentes a las convenciones nacionales de los partidas y un mayor control de los votantes sobre las nominaciones presidenciales. En efecto, como es sabido, en aquellos Estados en los que no se utiliza el procedimiento de las elecciones primarias, el sistema generalmente adoptado consiste en que los comités de los partidos estatales, es decir, varios reducidos grupos de personas -o caucusesseleccionen a los delegados, lo que indudablemente constituye un método menos democrático.

En contra de lo que cabía esperar teniendo presente el incremento del número de elecciones primarias y el hecho de que el porcentaje de la población en edad de votar en 1980 había aumentado aproximadamente en un 7 por 100 respecto de 1976 , el nivel de participación global de los ciudadanos no ha sido proporcionalmente superior. Más bien podría afirmarse lo contrario; en efecto, según la información aparecida en el Congressional Quartely Weekly Report de 5 de julio, tomando como punto de partida un total de 27 Estados en los que se celebraron elecciones

${ }^{1}$ Hasta el presente año no se habían celebrado elecciones primarias en Puerto Rico, Estado que, sin embargo, envía sus delegados a las convenciones de los partidos desde 1904 . Puerto Rico no es miembro de la Unión y sus ciudadanos no votan el día de la elección general. Puerto Rico no está, lógicamente, representado en el Congreso ni cuenta con voto electoral alguno.

2 En virtud de la aplicación de la enmienda 23 a la Constitución, aprobada en 1961, el distrito de Columbia, que carece de representación en el Congreso, cuenta con tres votos electorales. 
primarias en 1976 y 1980, resulta que la participación en las elecciones organizadas por el partido republicano aumentó en un 7,7 por 100 , mientras que en las organizadas por el partido demócrata sufrió una disminución del 5,7 por 100 .

Como puede observarse en los cuadros transcritos a continuación, los republicanos no celebraron elecciones en Arkansas y Nueva York ni los demócratas en Carolina del Sur. Este fenómeno encuentra su explicación en el hecho de que en el ámbito de los procedimientos de selección de los delegados para las convenciones nacionales de los partidos las normas aplicables no son las federales, sino que varían - cuando existen - en cada Estado y para cada partido.

Los resultados de las elecciones presidenciales primarias que aparecen reproducidos han sido extraídos del Congressional Quarterly Weekly Report, vol. 38, nú-

\section{DEMOCRATAS}

\begin{tabular}{|c|c|c|c|c|c|}
\hline & $\begin{array}{l}\text { Número } \\
\text { de } \\
\text { votantes }\end{array}$ & Brown & Cárter & Kennedy & $\begin{array}{l}\text { Sin } \\
\text { prefe- } \\
\text { rencia }\end{array}$ \\
\hline $\begin{array}{ccccccc}\text { Alabama } & \ldots & \ldots & \ldots & \ldots & \ldots & \ldots\end{array}$ & 237.464 & 4,0 & 81,6 & 13,2 & 0,7 \\
\hline $\begin{array}{ccccccc}\text { Arkansas } & \ldots & \ldots & \ldots & \ldots & \ldots & \ldots \\
\end{array}$ & 448.290 & - & 60,1 & 17,5 & 18,0 \\
\hline $\begin{array}{ccccccc}\text { California } & \ldots & \ldots & \ldots & \ldots & \ldots & \ldots \\
\end{array}$ & 3.323 .812 & $\overline{4,0}$ & 37,7 & 44,8 & 11,4 \\
\hline Carolina del Norte ... ... ... & 737.262 & 1,9 & 70,1 & 17,7 & 9,3 \\
\hline Connecticut $\ldots \ldots \ldots \ldots \ldots$ & 210.275 & 2,6 & 41,5 & 46,9 & 6,4 \\
\hline Dakota del Sur $\ldots . \ldots . .$. & 67.671 & - & 45,9 & 48,2 & 5,9 \\
\hline $\begin{array}{ccccccc}\text { Florida } & \ldots & \ldots & \ldots & \ldots & \ldots & \ldots\end{array}$ & 1.098 .003 & 4,9 & 60,7 & 23,2 & 9,5 \\
\hline 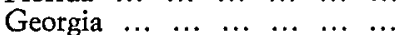 & 384.780 & 1,9 & 88,0 & 8,4 & 1,0 \\
\hline 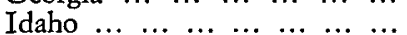 & 50.482 & 4,1 & 62,2 & 22,0 & 11,8 \\
\hline $\begin{array}{lllllll}\text { Illinois } & \ldots & \ldots & \ldots & \ldots & \ldots & \ldots\end{array}$ & 1.201 .067 & 3,3 & 65,0 & 30,0 & - \\
\hline 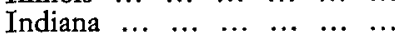 & 589.441 & - & 67,7 & 32,3 & - \\
\hline $\begin{array}{lllllll}\text { Kansas } & \ldots & \ldots & \ldots & \ldots & \ldots & \ldots\end{array}$ & 193.918 & 4,9 & 56,6 & 31,6 & 5,8 \\
\hline $\begin{array}{lllllllll} & \\
\text { Kentucky } & \ldots & \ldots & \ldots & \ldots & \ldots & \ldots & \ldots\end{array}$ & 240.331 & 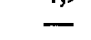 & 66,9 & 23,0 & 8,0 \\
\hline $\begin{array}{lllllll}\text { Louisiana } & \ldots & \ldots & \ldots & \ldots & \ldots & \ldots\end{array}$ & 358.741 & 4,7 & 55,7 & 22,5 & 11,6 \\
\hline $\begin{array}{lllllll}\text { Maryland } & \ldots & \ldots & \ldots & \ldots & \ldots & \ldots\end{array}$ & 477.090 & 3,0 & 47,5 & 38,0 & 9,6 \\
\hline 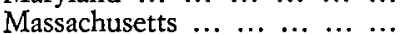 & 907.332 & 3,5 & 28,7 & 65,1 & 2,2 \\
\hline $\begin{array}{lllllll}\text { Michigan } & \ldots & \ldots & \ldots & \ldots & \ldots & \ldots\end{array}$ & 78.424 & 29,4 & - & - & 46,4 \\
\hline $\begin{array}{lllllll}\text { Montana } & \ldots & \ldots & \ldots & \ldots & \ldots & \ldots\end{array}$ & 125.002 & & 51,6 & 37,2 & 11,2 \\
\hline $\begin{array}{ccccccc}\text { Nebraska } & \ldots & \ldots & \ldots & \ldots & \ldots & \ldots\end{array}$ & 153.881 & 3,6 & 46,9 & 37,6 & 10,4 \\
\hline $\begin{array}{llllllll}\text { Nevada } & \ldots & \ldots & \ldots & \ldots & \ldots & \ldots & \ldots\end{array}$ & 66.948 & - & 37,6 & 28,8 & 33,6 \\
\hline Nueva Hampshire $\ldots . . . . . . .$. & 11.930 & 9,6 & 47,1 & 37,3 & - \\
\hline $\begin{array}{lllll}\text { Nueva Jersey } & \ldots & \ldots & \ldots & \ldots\end{array}$ & 560.908 & - & 37,9 & 56,2 & 3,5 \\
\hline 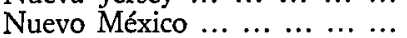 & 157.499 & 一 & 41,9 & 46,1 & 6,1 \\
\hline $\begin{array}{llllll}\text { Nueva York } & \ldots & \ldots & \ldots & \ldots & \ldots\end{array}$ & 989.062 & - & 41,1 & 58,9 & - \\
\hline $\begin{array}{ccccccc}\text { Ohio } & \ldots & \ldots & \ldots & \ldots & \ldots & \ldots\end{array}$ & 1.183 .499 & - & 51,0 & 44,1 & - \\
\hline Oregón $\ldots \ldots \ldots \ldots \ldots$ & 343.050 & 9,7 & 58,2 & 32,1 & - \\
\hline $\begin{array}{llllll} & \text { Pennsylvania } & \ldots & \ldots & \ldots & \ldots \\
\end{array}$ & 1.613 .223 & 2,3 & 45,4 & 45,7 & 5,8 \\
\hline Rhode Island $\ldots \ldots \ldots \ldots \ldots$ & 38.327 & 0,8 & 25,8 & 68,3 & 2,0 \\
\hline 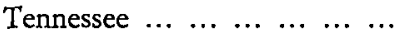 & 294.680 & 1,9 & 75,2 & 18,1 & 3,9 \\
\hline $\begin{array}{cccccccc}\text { Texas } & \ldots & \ldots & \ldots & \ldots & \ldots & \ldots & \ldots\end{array}$ & 1.377 .354 & 2,6 & 55,9 & 22,8 & 18,7 \\
\hline $\begin{array}{lllllll}\text { Vermont } & \ldots & \ldots & \ldots & \ldots & \ldots & \ldots\end{array}$ & 39.703 & 0,9 & 73,1 & 25,5 & $\underline{-12}$ \\
\hline Virginia Occidental $\ldots . . .$. & 314.985 & - & 61,9 & 38,1 & - \\
\hline $\begin{array}{lllllll} & W \text { isconsin } & \ldots & \ldots & \ldots & \ldots & \ldots\end{array}$ & 629.619 & 11,8 & 56,2 & 30,1 & 0,4 \\
\hline Distrito de Columbia ... ... & 64.150 & - & 36,9 & 61,7 & - \\
\hline $\begin{array}{llllll} & & & & & \\
\text { Puerto Rico } & \ldots & \ldots & \ldots & \ldots\end{array}$ & 870.235 & 0,2 & 51,7 & 48,0 & - \\
\hline $\begin{array}{llll}\text { Totales } & \ldots & \ldots & \ldots\end{array}$ & 19.538 .438 & 2,9 & 51,2 & 37,6 & 6,6 \\
\hline
\end{tabular}


mero 27 , de 5 de julio de 1980, y están basados —-según dicha publicación- en informes oficiales, a excepción de los correspondientes a California, Dakota del Sur, Montana, Nuevo México, Ohio, Oregón y Virginia Occidental. La única variación es que hemos alterado el orden allí establecido, que era el cronológico, sustituyéndolo por el alfabético para facilitar su más rápida consulta.

REPUBLICANOS

\begin{tabular}{|c|c|c|c|c|c|c|c|c|}
\hline & $\begin{array}{c}\text { Número } \\
\text { de } \\
\text { votantes }\end{array}$ & $\begin{array}{c}\text { An- } \\
\text { derson }\end{array}$ & Baker & Busch & $\begin{array}{l}\text { Con- } \\
\text { nally }\end{array}$ & Crane & Reagan & $\begin{array}{l}\text { Sin } \\
\text { prefe- } \\
\text { rencia }\end{array}$ \\
\hline Alabama & 211.353 & 一 & 0,9 & 25,9 & 0,5 & 2,4 & 69,7 & - \\
\hline $\begin{array}{cccc}\text { California } & \ldots & \ldots & \ldots\end{array}$ & 2.512 .994 & 13,6 & - & 4,9 & - & 0,9 & 80,2 & - \\
\hline Carolina del Norte. & 168.391 & 5,1 & 1,5 & 21,8 & 0,7 & 0,3 & 67,6 & 2,7 \\
\hline Carolina del Sur ... & 145.501 & - & 0,5 & 14,8 & 14,8 & 19,6 & 54,7 & - \\
\hline $\begin{array}{lll}\text { Connecticut } & \ldots & \ldots\end{array}$ & 82.284 & 22,1 & 1,3 & 38,6 & 0,3 & 1,0 & 33,9 & 2,3 \\
\hline Dakota del Sur ... & 88.325 & 6,3 & - & 4,2 & - & 0,5 & 82,1 & 5,8 \\
\hline Florida $\ldots \ldots \ldots \ldots$ & 614.995 & 9,2 & 1,0 & 30,2 & 0,8 & 2,0 & 56,2 & - \\
\hline Georgia $\ldots \ldots \ldots \ldots$ & 200.171 & 8,4 & 0,8 & 12,6 & 1,2 & 3,2 & 73,2 & - \\
\hline $\begin{array}{llllll}\text { Idaho } & \ldots & \ldots & \ldots & \ldots\end{array}$ & 134.879 & 9,7 & - & 4,0 & - & 0,8 & 82,9 & 2,6 \\
\hline $\begin{array}{lllll}\text { Illlinois } & \ldots & \ldots & \ldots & \ldots\end{array}$ & 1.130 .081 & 36,7 & 0,6 & 11,0 & 0,4 & 2,2 & 48,4 & - \\
\hline $\operatorname{Indiana} \ldots \ldots \ldots$ & 568.315 & 9,9 & - & 16,4 & - & - & 73,7 & - \\
\hline $\begin{array}{ccccc}\text { Kansas } & \ldots & \ldots & \ldots & \ldots\end{array}$ & 285.398 & 18,2 & 1,3 & 12,6 & 0,7 & 0,5 & 63,0 & 2,4 \\
\hline $\begin{array}{cccc}\text { Kentucky } & \ldots & \ldots & \ldots\end{array}$ & 94.795 & 5,1 & - & 7,2 & - & - & 82,4 & 3,3 \\
\hline $\begin{array}{lllll}\text { Louisiana } & \ldots & \ldots & \ldots\end{array}$ & 41.683 & - & - & 18,8 & - & - & 74,9 & 5,3 \\
\hline $\begin{array}{lllll}\text { Maryland } & \ldots & \ldots & \ldots\end{array}$ & 167.303 & 9,7 & - & 40,9 & - & 1,3 & 48,2 & - \\
\hline Massachusetts $\ldots \ldots$ & 400.826 & 30,7 & 4,8 & 31,0 & 1,2 & 1,2 & 28,8 & 0,6 \\
\hline $\begin{array}{lllll}\text { Michigan } & \ldots & \ldots & \ldots\end{array}$ & 595.176 & 8,2 & - & 57,5 & - & - & 31,8 & 1,7 \\
\hline $\begin{array}{cccc}\text { Montana } & \ldots & \ldots & \ldots\end{array}$ & 76.716 & - & - & 9,7 & - & - & 87,3 & 3,0 \\
\hline $\begin{array}{cccc}\text { Nebraska } & \ldots & \ldots & \ldots\end{array}$ & 205.203 & 5,8 & - & 15,3 & - & 0,5 & 76,0 & - \\
\hline Nevada $\ldots \ldots \ldots \ldots$ & 47.395 & - & - & 65 & - & - & 83,0 & 10,5 \\
\hline Nueva Hampshire ... & 147.157 & 9,8 & 12,9 & 22,7 & 1,5 & 1,8 & 49,6 & - \\
\hline Nueva Jersey $\ldots . .$. & 277.977 & - & - & 17,1 & - & - & 81,3 & - \\
\hline Nuevo México .... ... & 59.101 & 12,1 & 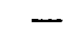 & 9,9 & - & 7,5 & 63,7 & 2,2 \\
\hline Ohio $\ldots \ldots \ldots \ldots \ldots$ & 854.967 & - & - & 19,2 & - & - & 80,8 & - \\
\hline Oregón $\ldots \ldots \ldots \ldots$ & 304.647 & 10,1 & - & 34,7 & - & 0,7 & 54,5 & - \\
\hline $\begin{array}{llll}\text { Pennsylvania } & \ldots & \ldots\end{array}$ & 1.241 .002 & 2,1 & 2,5 & 50,5 & 0,9 & - & 42,5 & - \\
\hline Rhode Island $\ldots . .$. & 5.335 & - & - & 18,6 & - & - & 72,0 & 6,5 \\
\hline Tennessee $\ldots \ldots \ldots$ & 195.210 & 4,5 & - & 18,1 & - & 0,8 & 74,1 & 2,5 \\
\hline $\begin{array}{llllll}\text { Texas } & \ldots & \ldots & \ldots & \ldots\end{array}$ & 526.769 & - & - & 47,4 & - & - & 51,0 & 1,5 \\
\hline $\begin{array}{llll}\text { Vermont } & \ldots & \ldots & \ldots\end{array}$ & 65.611 & 29,0 & 12,3 & 21,7 & 1,3 & 1,9 & 30,1 & - \\
\hline Virginia Occ. $\ldots . .$. & 133.871 & - & - & 14,4 & - & - & 85,6 & - \\
\hline Wisconsin $\ldots \ldots \ldots$ & 907.853 & 27,4 & 0,4 & 30,4 & 0,3 & 0,3 & 40,2 & 0,3 \\
\hline Distr. de Columbia. & 7.529 & 26,9 & & 66,1 & - & 3,6 & - & - \\
\hline Puerto Rico ... ... . ... & 186.371 & - & 37,0 & 60,1 & 1,1 & - & - & - \\
\hline Totales ... ... & 12.785 .184 & 12,3 & 1,4 & 24,0 & 0,6 & 0,8 & 59,7 & 0,5 \\
\hline
\end{tabular}

